\title{
Psicología en deporte universitario: estudio de carga mental y habilidades psicológicas en deporte de alto rendimiento'
}

\author{
Juan Camilo Martínez Juan Alejandro Pérez \\ Fernández* Quintero**
}

Artículo corto de investigación recibido el 07/02/2018

y aprobado el 10/03/2018

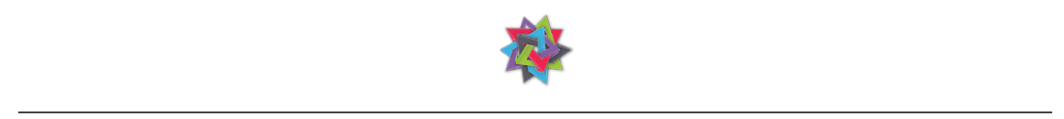

Cómo citar este artículo:

Martínez Fernández, J., \& Pérez Quintero, J. (2018). Psicología en deporte universitario: estudio de carga mental y habilidades psicológicas en deporte de alto rendimiento. Trans-Pasando Fronteras, (11).

\footnotetext{
${ }^{1}$ Artículo resultado de la iniciativa investigativa de los autores como exalumno y alumno de la universidad, y como deportistas de alto rendimiento universitario.

* Psicólogo de la Universidad Icesi (Cali, Colombia)

**Estudiante de Psicología de la Universidad Icesi (Cali, Colombia)
} 


\section{Resumen}

La presente investigación tiene como propósito exponer y describir los niveles de carga mental y de diferentes habilidades psicológicas relacionadas con el rendimiento deportivo en deportistas de alto rendimiento de la Universidad Icesi de la ciudad de Santiago de Cali (Colombia). Se efectuó una investigación no experimental de tipo transversal en la que se aplicaron los cuestionarios NASA-TLX y CPRD a 39 deportistas (24 hombres y 15 mujeres), entre edades de 18 años a 24 años. Los resultados del NASA-TLX dieron evidencia de un nivel de carga mental alto en los sujetos, lo que sugiere que la exigencia académica combinada con el ejercicio voluntario de deporte, Ilevan a los atletas universitarios a unos niveles peligrosos de esfuerzo mental. Por otro lado, los puntajes obtenidos tras la aplicación del CPRD muestran que hay evidencia de diferencias significativas en los niveles de motivación mostrados por los deportistas que practican deportes individuales frente a los que practican deportes de conjunto, que pueden tener causa en las maneras en cómo se relacionan los deportistas. Estos resultados sugieren la pertinencia de la participación de la psicología en el entorno deportivo para lograr un entrenamiento íntegro, en el que se tenga en la cuenta esas diferencias y así construir un ambiente óptimo para el buen desarrollo personal, emocional y, claramente, deportivo.

Palabras clave: Carga mental; Deporte; Alto rendimiento; Habilidad psicológica; Psicología del deporte. 


\section{Psychology in college sport: study of mental load and psychological skills in high performance sport}

\section{Abstract}

The present investigation has the purpose to expose the levels of mental load and of different psychological abilities related to the athletic performance in athletes of high performance of the Icesi University of Santiago de Cali (Colombia). A non-experimental cross-sectional investigation was carried out in which the NASA-TLX and CPRD questionnaires were applied to 39 athletes (24 men and 15 women), between the ages of 18 and 24 years old.

The results of the NASA-TLX gave evidence of a high level of mental load in the subjects, which suggests that the academic as well as the sporting demand of the university is taking the university athletes to dangerous levels of mental effort. On the other hand, the scores obtained after the application of CPRD show that there is evidence of significant differences in motivation between individual and joint sports, which may have cause in the ways in which athletes relate to each other. These results suggest the relevance of the participation of psychology in the sports environment to achieve an integral training, in which those differences are considered and thus build an optimal environment for good personal, emotional and, clearly, sports development.

Keywords: Mental load; Sport; High performance; Psychological ability; Sports psychology. 


\section{Introducción}

Este documento surge -en el marco de la Psicología Deportiva- como una propuesta de investigación y labor profesional, con el fin de demostrar tres aspectos relevantes: primero, la inmersión de la psicología del deporte como una nueva y establecida disciplina en Colombia; en segundo lugar, los aportes desde la academia para el desarrollo conceptual del trabajo, el cual cuenta con sustentación teórica desde las áreas: educativa, social, organizacional, psicoanálisis, desarrollo, procesos e histórico-cultural; por último, pero no menos importante, el buscar que la visión de sujeto dentro del deporte sea restablecida como un ser humano, y no un objeto de valor intercambiable.

Este artículo presenta los resultados de una investigación para generar una propuesta de un plan de entrenamiento psicológico para deportistas de alto rendimiento deportivo en contextos universitarios, lo cual busca lograr un rendimiento óptimo, a través del bienestar emocional y psíquico de los deportistas estudiantiles. La idea central parte de lo observado en las selecciones representativas deportivas de la universidad, y el cómo se ha hecho evidente que el rendimiento en las competencias en las que participan tiene un margen de mejoramiento ${ }^{2}$. Es por ello, que el sentido de pertenencia invita a proponer esta investigación con soporte de estudios realizados durante los semestres académicos y experiencias profesionales. Este estudio busca incorporarse en el modelo de entrenamiento y planificación del área de Recreación y Deporte de Bienestar Universitario, con el fin de consolidarse como una parte fundamental del proceso deportivo de los estu-

${ }^{2}$ Los gestores de este proyecto han participado como deportistas y como espectadores del proceso deportivo en la universidad, especialmente 
diantes, entrenadores y demás participantes.

Antes de mostrar la propuesta completa, se hace necesario responder a una serie de preguntas que, según los expertos en Psicología del Deporte, surgen al momento de presentar un plan de entrenamiento psicológico, siendo las más importantes ¿Por qué debería incorporar un plan de entrenamiento psicológico? ¿Qué beneficios y qué falencias trae consigo implementarlo? ¿Cómo se estructura un plan de entrenamiento psicológico a un plan deportivo ya establecido?, todas se responderán con el mínimo de palabras posibles, ya que pretende hacer un recorrido histórico corto por la psicología deportiva, para después enfocarse en la presentación del concepto de plan de entrenamiento psicológico.

Entrando en materia, la historia de la psicología del deporte en Colombia se divide en cuatro períodos (1997-1979/1980-1989/ 1990-1999/ 2000-Actualidad) según Humberto Serrato (2008), los cuales se hacen relevantes e importantes desde 1973, donde se empieza a oficializar el concepto de Psicología del deporte como una rama disciplinar dentro de la ciencia psicológica, y a partir de allí vienen una serie de eventos que ayudan a fortalecer, desarrollar y consolidar a la Psicología del deporte como una parte esencial dentro de las ciencias del deporte, el deporte nacional e incluso el deporte olímpico. Tanto así, que, en las olimpiadas de 2014, realizadas en Rio de Janeiro (Brasil), Colombia contó con seis psicólogos especializados en esta área, para apoyar y entrenar en la villa olímpica a los representantes del país, cuando antes si mucho se pensaba para llevar a uno³.

${ }^{3}$ Datos obtenidos de Semana.com, Articulo "La ciencia detrás de la mentalidad ganadora de los colombianos en río", Oskar Antonio Ortiz Echeverría, Colaborador. Consultado el 20/09/2017 
Los eventos en cuestión por mencionar los más importantes son: la incorporación de los psicólogos bogotanos Esther Granados y Edison Ramos en Coldeportes Nacional, quienes por décadas motivaron a la Psicología colombiana para que se vinculara al proceso deportivo, llegando así a otro evento significativo, y uno de los más recientes, el cual ocurrió en la primera década del nuevo milenio donde se inicia con la creación de la especialización en psicología del deporte en la Universidad El Bosque de Bogotá; actualmente, codirigido por Sandra Y. García y María Clara Rodríguez, quienes en su labor, hasta el día de hoy, han capacitado a más de 100 especialistas. Paralelo a todo esto, se han venido realizando un elevado número de simposios, conversatorios y congresos nacionales e internacionales dentro de Colombia, el más pronto, fue el VI Congreso Nacional e Internacional de Psicología del Deporte, que tuvo lugar en Bogotá en la universidad INCCA, contando con la participación y apoyo de la Sociedad Iberoamericana de Psicología del Deporte (SiPD), que bien ha venido incorporando Psicólogos profesionales colombianos, como por ejemplo el mismo Humberto Serrato, quien oficia como representante de la SiPD en Colombia.

En esos eventos, se han venido presentando y exponiendo investigaciones, tesis, proyectos y planes, que demuestran la efectividad y la necesidad de esta área donde quiera que haya deportistas. Serrano explica bien las desventajas de todo esto, diciendo que "después de 35 años el balance de investigaciones y publicaciones es pobre. Este es un aspecto en el que la psicología del deporte colombiana debe progresar" (Serrano, 2008, p. 297). Por eso, este documento se apoya sobre la petición del autor para hacer necesaria la difusión de los logros académicos y prácticos de los profesionales, incluyendo investigaciones, proyectos, entre otros modos. 
Ahora bien, que ya se contextualizó a la Psicología del deporte, es momento de profundizar en el porqué del plan de entrenamiento psicológico. "Mens sana in corpore sano» ("Mente sana en un cuerpo sano") dice el texto Sátiras de Juvenal (Roma Imperial), usado como un lema por múltiples equipos de distintas disciplinas. De esa frase se asume que la Psicología del deporte ha podido consolidarse como una rama académica importante en los últimos años. En la actualidad, se vive en una sociedad que rinde un considerable culto al cuerpo. La ropa deportiva, los productos dietéticos y de la belleza, los suplementos alimenticios, los gimnasios y centros deportivos, etc., forman parte de una industria. Además, los atletas y deportistas de élite gozan de una relevante fama y admiración. Parece indiscutible que esta tendencia por gozar de una buena salud en el plano físico es una buena noticia, pero ¿qué ocurre con nuestra mente? Esta ciencia interdisciplinar pretende dar respuestas a algunas necesidades en el mundo del deporte a) Proporcionar asistencia psicológica a atletas, equipos, así como asesoramiento a entrenadores, preparadores físicos y docentes. b) Optimizar los recursos personales del deportista y contribuir en el progreso de la calidad del manejo táctico y de comunicación. c) Desarrollar técnicas para el entrenamiento de habilidades mentales como la concentración, gestión de estrés y ansiedad.

No solo se logra mencionar varios beneficios de un entrenamiento psicológico, sino que, con esa característica interdisciplinar, esta rama psicológica toma elementos de la psicología, la fisiología, la kinesiología, la sociología y la biomecánica, y cuyo objeto es el estudio de cómo los factores psicológicos afectan al desempeño deportivo y cómo la participación en el deporte y el ejercicio influye en el desarrollo psicológico y el estado físico. Es por esto que se estudia, se analiza y se interviene en la relación cuerpo-mente; García (2006) expone que todos los seres vivos, especialmente el ser hu- 
mano, al experimentar cualquier emoción en el cuerpo, genera una serie de respuestas como la segregación de sudor, aumento de la frecuencia cardíaca y respiratoria, tensión muscular, etc., y que es debido a esta afectación directa en el organismo, originada por los estados emocionales, que se puede concluir que tan importante es entrenar el cuerpo en conjunto con la mente.

Por lo anterior, se va haciendo evidente el porqué de un entrenamiento psicológico, y es que, si se suman las variables que rodean a un estudiante que practica deporte, tales como: presión de rendimiento académico (por becas o familiar), estrés por exámenes o quices, relaciones interpersonales y demás, hace necesario el acompañamiento psicológico para que el deportista sienta un apoyo más personal y enfocado a lo que ocurre en su mente. El propósito central del entrenamiento psicológico es incidir sobre los determinantes psicológicos de las acciones, con la intención de optimizar la ejecución técnico-táctica del deportista. Este proceso, al igual que todo entrenamiento, implica la consideración de al menos tres componentes: i) Determinar y valorar el perfil psicológico del deportista en relación con la práctica de un deporte específico; situación que implica la identificación de las condiciones y características actuales del deportista (estado es). ii) Identificar las destrezas psicológicas que requiere el deportista, para lograr un rendimiento máximo en la práctica de un deporte específico. Es decir, la definición operativa del estado al que se quiere llevar deportista (estado debe ser). iii) Establecer la estrategia metodológica a seguir en la transición del estado del deber ser. Al respecto es preciso empoderarse de los conocimientos y técnicas precisas, para promover el desarrollo y consolidación de las destrezas psicológicas necesarias para la práctica exitosa de un deporte específico. 
Los componentes están rodeados un sin fin de habilidades psicológicas que son particulares de cualquier interacción humana, pero los expertos en este tipo de estudios y trabajo, como por ejemplo Ureña (2003), y concuerda Balagué et al (1997), diciendo que en el deporte se estudian y evalúan: el desarrollo de la percepción, el almacenaje de información, la forma de pensar, la toma de decisiones, la imaginación, la memoria, las habilidades comunicativas, la atención, la motivación, el manejo de estrés y el manejo de emociones positivas y negativas. Por lo cual, según Matveiev (1977) citado por González (1992, p. 225), "[...]toda planificación se puede considerar como un sistema de establecimiento de metas, consistente en la anticipación de una actividad que se pretende llevar a la práctica; en el caso de la planificación deportiva, será un proyecto del contenido, de las formas y condiciones del entrenamiento". Se trata -según el autor- de un proyecto mental que se elabora antes de que tenga lugar su inserción en el entrenamiento deportivo. La planificación del entrenamiento psicológico persigue el mismo objetivo, programar de antemano un plan que después se intentará hacer realidad, con el fin de optimizar el rendimiento deportivo.

Este proceso constituye un procedimiento más, que se integra dentro de la planificación global. La planificación de este entrenamiento psicológico se integra en el Plan general de entrenamiento y se adapta a sus fases, contribuyendo así a la consecución de los mejores resultados, mediante la preparación mental del deportista. Puesto que la dimensión psicológica no es un aspecto del cual el deportista puede prescindir cuando compite, el entrenamiento mental se planifica en plena correspondencia con el entrenamiento físico. Así pues, se pueden desprender múltiples limitaciones o falencias de este entrenamiento. En palabras de Garcés de Los Fayos y Vives (2005), las limitaciones de la planificación de un entrena- 
miento psicológico se agrupan en cuatro áreas: profesional, personal, deportiva y social, la escuela o universidad se incluye en esta última área. En resumen, dicen que por lo profesional se presenta que haya: ausencia de formación suficiente en psicología del deporte, desconocimiento técnico, táctico y físico del deporte y grandes dificultades comunicativas entre los diferentes profesionales del deporte. En el área personal: ausencia de competencias sociales y personales generales, la asunción de discriminación objetiva debida al sexo y asumir el alto nivel competitivo en el trabajo a realizar. Por el lado del área deportiva se pueden presentar: falta de entendimiento con otros profesionales del deporte, dificultades para estar presente en los entrenamientos y en las competiciones, no aceptación del psicólogo del deporte por parte de los organismos deportivos y necesidad de una exclusividad absoluta.

Por último, pero no menos importante, está el área social, con limitaciones por: influencia negativa de familiares, incompatibilidad del deporte con el resto de los contextos vitales, existencia de intentos sistemáticos de controlar la vida de los deportistas, dificultad para diferenciar deporte profesional y deporte recreativo, dificultad para vincular la vida académica y presión incontrolable de las personas que rodean al deportista.

Garcés de Los Fayos \& Vives (2005) sugieren que, para evitar esas limitaciones, el profesional debe estar lo mejor preparado posible para aplicar un plan de entrenamiento, con el fin de no caer en juicios de valor que lo hagan parecer otra área psicológica que no tenga que ver con el campo deportivo, pues, aunque este campo reúna muchos factores de las demás ramas, es importante delimitar funciones y estrategias. De igual forma, ellos solicitan que la comunicación con los demás involucrados como entrenadores, administrativos, familiares, etc., sea permanente y que se 
aclaren desde el principio los roles que cada uno va a desempeñar en el entrenamiento, pues como se dijo, lo psicológico va subyacente a lo físico-táctico-técnico, y es el entrenador quien dispone u otorga el tiempo para que el psicólogo pueda intervenir. Y de eso último, se desprende la más importante sugerencia, y es que el plan de entrenamiento mental debe buscar siempre ser flexible para acondicionarse al plan macro que se establece para el fortalecimiento táctico-técnico, teniendo en cuenta los ciclos en lo que se maneja.

Por ello, el objetivo principal de este esta investigación es desarrollar un plan de entrenamiento psicológico a deportistas que representan a la Universidad Icesi, para el mejoramiento del rendimiento deportivo; a partir de este, se busca: primero, potenciar las habilidades psicológicas como Autoconfianza, Motivación, Procesos Cognitivos, Control de las habilidades emocionales y Manejo de las habilidades sociales contribuyendo a un nivel óptimo de rendimiento académico. Segundo, brindar un nivel óptimo de bienestar psicológico, teniendo en cuenta los valores representativos de Bienestar Universitario y la Universidad Icesi. Y tercero, pero no menos importante, se espera fortalecer la cultura universitaria de los estudiantes y el personal de apoyo involucrado en el marco interno y externo de este proyecto.

Es por todo ello que la hipótesis base para esta investigación es que no hay evidencia de que las cargas mentales de los deportistas de este estudio, y sus habilidades mentales, sean semejantes. De los estudios de García (2006) se puede inferir que los deportes de conjunto manejan un bajo nivel de exigencia (carga) mental que los deportes individuales, teniendo mejor desarrollo de sus habilidades psicológicas. Por tanto, para los análisis estadísticos, la hipótesis nula (Ho) es: los promedios de carga mental y de habi- 
lidades psicológicas en el deporte son iguales entre las diferentes muestras. Mientras que la hipótesis alternativa $(\mathrm{Ha})$ es: los promedios de carga mental y de habilidades psicológicas en el deporte no son iguales entre las diferentes muestras.

Ahora bien, a continuación, se presenta el plan que se busca desarrollar en la Universidad Icesi. Cabe anotar que este plan sigue los lineamientos públicos establecidos en los años 2015 por Coldeportes Nacional, ente reglamentario de la actividad profesional de las ciencias vinculadas al deporte.

\section{Metodología}

Este estudio se ubica dentro de la investigación no experimental, usando un diseño transaccional, los cuales tienen como objetivo describir relaciones entre dos o más variables en un momento determinado. Ese momento mencionado ocurre en los entrenamientos de 39 deportistas/estudiantes de alto rendimiento deportivo universitario, ya que es el ambiente indicado para poder acercarse a estos y evaluarlos.

Los sujetos que se eligen para la recolección de datos son los deportistas que hacen parte de las selecciones o equipos representativos de la Universidad Icesi, que además Ileven una práctica deportiva superior a un año calendario, y específicamente sean mayores de edad. La variable de años de práctica deportiva influye en el sentido de la adherencia y experticia del deportista, que, para ser considerado de alto rendimiento, es un factor importante. Dentro de las limitaciones del estudio se encuentra el tiempo y la disposición de los entrenadores, pues se inicia la investigación cuando el semestre ya ha avanzado, y, además, porque no son solo 
deportistas, sino estudiantes con responsabilidades académicas no se podría hacer una mayor recolección de datos con más líderes.

Se aplicarán los instrumentos Cuestionario NASA TLX diseñado por Arquer y Nogareda (2000). Cuyo objetivo es indagar ciertos aspectos respecto de la carga mental, física, rendimiento, exigencias temporales, nivel de frustración y esfuerzo que implica la realización de una tarea específica, haciendo énfasis en la recolección de datos. En adición al Cuestionario CPRD diseñado por Gimeno, Buceta y Pérez (2001). El cuestionario está compuesto por seis escalas: ansiedad (control de la ansiedad), autoconfianza, concentración, motivación, preparación mental y cohesión de equipo, por medio de las cuales se aporta información específica sobre las necesidades y recursos de los deportistas de competición. Los resultados serán analizados en el programa estadístico SPSS, con el fin de tener un análisis no paramétrico, que evidencia la correlación de las variables estudiadas mientras los deportistas iniciaban o terminaban su práctica deportiva.

Así pues, se contactará a los directivos del área de deportes de Bienestar Universitario mediante una carta de presentación de la investigación, para que evalúen la propuesta de trabajo y manifieste la pertinencia e idoneidad del estudio. Paso siguiente, se espera que se haga el llamado a los entrenadores de los deportes seleccionados, para que entonces, por común acuerdo, se organice la agenda de visitas para la aplicación de los cuestionarios, según el día y el tiempo disponible. A los directivos, entrenadores y deportistas se les presenta el documento de consentimiento informado para participar y se les explica el uso que después se hará de los resultados obtenidos. 


\section{Resultados}

Después de aplicar los cuestionarios a los 39 deportistas -de los cuales 24 son hombres y 15 son mujeres- con un rango de edad que va de los 18 a los 24 años, y con un rango de práctica y experiencia deportiva de 2 a 18 años; los resultados generales de esta investigación, se exponen en la tabla 1 a continuación, donde se presentan las medias obtenidas en cuanto a carga mental y las habilidades psicológicas en el rendimiento deportivo que analizan los cuestionarios NASA-TLX y CPRD, respectivamente. Los análisis que se hacen son tomando en cuenta las tablas que se muestran en los anexos, las cuales pertenecen a las demarcaciones de los cuestionarios originales.

Esta tabla muestra que en cuanto a la carga mental que poseen los deportistas, estos poseen un nivel muy alto de esta. Por el lado de las habilidades psicológicas de rendimiento deportivo, se encuentra que los deportistas en general tienen niveles casi óptimos

\begin{tabular}{|c|c|}
\hline Variable & Media \\
\hline Carga mental & 1273,6 \\
\hline Control de estrés & 50,8 \\
\hline $\begin{array}{c}\text { Influencia de la evaluación del rendimiento } \\
\text { deportivo }\end{array}$ & 28,6 \\
\hline Motivación & 21,7 \\
\hline
\end{tabular}


en cuanto a control de estrés, influencia de la evaluación, motivación, habilidad mental y cohesión de equipo se refiere. Esto en resumidas palabras, revela que los deportistas requieren de un muy alto "esfuerzo deliberado para alcanzar un resultado esperado". Lo negativo de este resultado, es que puede llegar a suceder el caso de que estos se "quemen", considerando la posible presencia del síndrome de Burnout.

Se presenta el segundo resultado pertinente, el cual es considerado de los datos que se subieron al programa de análisis estadístico SPSS, usando la prueba HSD de Tukey, la cual sirve para comparar las medias de los diferentes t niveles, en este caso, de las habilidades psicológicas deportivas en cada deporte, con el fin de especificar cualquier hipótesis alternativa genérica a parte de las que se consideran en las pruebas ANOVA. Este test ha arrojado las siguientes tablas que evidencian una diferencia significativa en el aspecto psicológico de la Motivación que estudia el Cuestionario de Características Psicológicas de rendimiento deportivo (CPRD).

Los resultados de la ANOVA señalaron que existen diferencias significativas entre las medias de los grupos en consideración ( $\mathrm{Na}$ tación y Baloncesto); $F(5,33)=2,57$; en particular las pruebas post hoc mostraron que en los nadadores tienen niveles de motivación menor que las personas que practican baloncesto esto significa que al menos para esta muestra, las personas que practican deportes individuales aparentemente tendrían menos nivel de motivación que las personas que practican deportes de conjunto. 


\begin{tabular}{|l|cc|c|l|l|}
\hline Deporte & N & $\begin{array}{l}\text { X (media } \\
\text { muestral) }\end{array}$ & $\begin{array}{l}\text { S (desviación } \\
\text { estándar) }\end{array}$ & F (5,33) & Post hoc \\
\hline 1.Natación & 3 & 17,33 & 0,94 & $2,57^{*}$ & $1 / 6$ \\
\hline 2. Rugby & 6 & 19,50 & 4,31 & & \\
\hline 3. Fútbol & 10 & 20,60 & 4,76 & & \\
\hline 4. Tenis de Mesa & 5 & 20,80 & 4,58 & & \\
\hline 5.Voleibol & 10 & 24,00 & 3,63 & & \\
\hline 6. Baloncesto & 5 & 25,60 & 1,74 & & \\
\hline
\end{tabular}

Subconjunto para alfa $=0.05 * \mathbf{P}<0,05$

Tabla 2 - Motivación por deportes

\begin{tabular}{|c|c|c|}
\hline \multicolumn{2}{|c|}{ Deporte $\mathbf{N}$} & \multirow{2}{*}{ 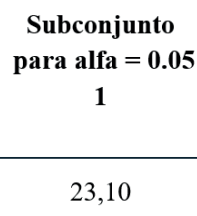 } \\
\hline Fútbol & 10 & \\
\hline Natación & 3 & 25,00 \\
\hline Rugby & 6 & 25,17 \\
\hline Tenis de mesa & 5 & 27,00 \\
\hline Baloncesto & 5 & 32,40 \\
\hline Voleibol & 10 & 36,20 \\
\hline Sig. & &, 258 \\
\hline
\end{tabular}

Tabla 3 - Influencia de la evaluación del rendimiento deportivo 
Psicología en deporte univesitario: Estudio de carga mental y habilidades psicológicas

\begin{tabular}{|c|c|c|c|}
\hline \multirow{2}{*}{ Deporte } & & \multicolumn{2}{|c|}{ Subconjunto para alfa $=\mathbf{0 . 0 5}$} \\
\hline & & 1 & 2 \\
\hline Natación & 3 & 17,33 & \\
\hline Rugby & 6 & 19,50 & 19,50 \\
\hline Fútbol & 10 & 20,60 & 20,60 \\
\hline Tenis de mesa & 5 & 20,80 & 20,80 \\
\hline Voleibol & 10 & 24,00 & 24,00 \\
\hline Baloncesto & 5 & & 25,60 \\
\hline Sig. & &, 126 & ,194 \\
\hline
\end{tabular}

Tabla 4-Motivación

Se visualizan las medias para los grupos en los subconjuntos homogéneos.

\begin{tabular}{|c|c|c|}
\hline \multicolumn{2}{|c|}{ Deporte N } & $\begin{array}{c}\text { Subconjunto } \\
\text { para alfa }=\mathbf{0 . 0 5} \\
\text { 1 }\end{array}$ \\
\hline Tenis de mesa & 5 & 18,00 \\
\hline Baloncesto & 5 & 20,80 \\
\hline Fútbol & 10 & 22,20 \\
\hline Natación & 3 & 22,33 \\
\hline Rugby & 6 & 23,17 \\
\hline Voleibol & 10 & 23,80 \\
\hline Sig. & &, 673 \\
\hline
\end{tabular}

Tabla 5 - Habilidad Mental 


\begin{tabular}{|c|c|c|}
\hline \multicolumn{2}{|c|}{ Deporte N } & \multirow{2}{*}{$\begin{array}{c}\begin{array}{c}\text { Subconjunto } \\
\text { para alfa }=\mathbf{0 . 0 5} \\
\mathbf{1}\end{array} \\
15,00\end{array}$} \\
\hline Natación & 3 & \\
\hline Rugby & 6 & 17,33 \\
\hline Tenis de mesa & 5 & 18,00 \\
\hline Fútbol & 10 & 19,50 \\
\hline Baloncesto & 5 & 20,60 \\
\hline Voleibol & 10 & 20,80 \\
\hline Sig. & & 067 \\
\hline
\end{tabular}

De acuerdo con los datos mostrados en las tablas 2 a la 5, es pertinente decir para un nivel de homogeneidad de varianza de 0,05; los 39 deportistas de las selecciones representativas de la Universidad Icesi muestran que en cuanto a las habilidades deportivas que analiza el CPRD, no hay diferencias significativas en cuanto a la Influencia de evaluación, la habilidad mental y la cohesión de equipo, a pesar de intervenir en deportes considerados individuales (natación y tenis de mesa). Sin embargo, el test HSD de Tukey, enseña que en el aspecto de motivación sí hay diferencias significativas entre los deportes individuales y de conjunto, como el caso entre natación y baloncesto. Este análisis surge a partir de que en el test HSD genera dos columnas para que sea visible esa diferencia entre deportes según la habilidad, y como se puede 
notar en las otras habilidades la columna dos no se da. Con ello, es evidente que la habilidad psicológica de motivación parece experimentarse de manera diferente en los deportes de conjunto e individuales.

\section{Discusión}

Tal y como lo menciona Gabriel Casarico en su artículo "Motivando a un equipo": "[...] en cualquier ámbito donde se encuentren las personas sea en una la empresa, el equipo deportivo, la escuela, para crecer es importante cierta disposición mental [...]", ésa es la necesidad de hacer algo hoy mejor que ayer según como se puede interpretar. Esto implica observar, estar atento, concentrado y motivado por modificar el entorno. En este universo agitado de vertiginosos cambios es un desafío ineludible el de renovarse y reinventarse todo el tiempo, implicando esto diversos modos de encarar la realidad y tomar decisiones. Los cambios de las relaciones, las condiciones sociales, las reglas del juego requieren de un cambio en el modo de actuar del psicólogo y esta investigación así lo muestra. En este orden de ideas y debido a lo estudiado por Nieto y OImedilla (2001), en donde exploran la importancia de un plan de entrenamiento para mejorar el rendimiento en equipo de marcha universitario, se ha notado la relación entre la carga mental y las habilidades psicológicas, en donde se ve la necesidad de disminuir la carga mental elevada de los deportistas para que sus habilidades se potencien en el aspecto técnico y táctico. Así pues, se lograría una estabilidad en la motivación, la cual traería beneficios tanto a los procesos deportivos como académicos, pues ese equilibrio buscado, permite al deportista reconocer mejor sus estados de ánimo, los momentos de estrés y sus propias habilidades (y las de sus compañeros dado el caso) deportivas y personales. 


\section{Conclusiones}

Lo que sugieren los datos expuestos es que la exigencia académica combinada con el ejercicio voluntario de deporte, Ilevan a los atletas universitarios a unos niveles peligrosos de esfuerzo mental. Hay evidencia de lo dicho, gracias a los resultados de la ANO$V A$, pues muestran que hay diferencias significativas entre las medias de los grupos en consideración (Natación y Baloncesto), para así determinar que la hipótesis alternativa se cumple, ya que los nadadores tienen niveles más bajos de motivación que las personas que practican baloncesto, esto quiere decir que, para este muestra al menos, aquellos que practican deportes individuales superficialmente tienen menos nivel de motivación que aquellas personas que practican deportes de conjunto.

De acuerdo con lo anterior, y según Pons \& Puig (2004), "[...] si la carga mental es demasiado intensa y/o prolongada, puede causar a corto plazo situaciones de sobrecarga (fatiga) y subcarga (monotonía), y a largo plazo problemas de estrés [...]". Así pues, estos autores argumentan que la fatiga puede comportar situaciones de peligro para la persona. Pons \& Puig (2004) dicen que generalmente se perciben tres elementos de forma progresiva: quejas físicas, debilitamiento de los procesos cognitivos y motivacionales, y somnolencia o sopor. Estos elementos sugieren la necesidad de una intervención psicológica, en lo esencial integral, desde las áreas clínica-deportiva-educativa, con el fin de evitar la posible presencia del síndrome de Burnout y para garantizar un bienestar psicológico de los deportistas. De igual forma, permitiría esclarecer, si la carga tiene relación con la deserción deportiva y el rendimiento inconstante de los deportistas. 
Por otro lado, el estudio muestra que hay evidencias de diferencias significativas en la motivación entre deportes individuales y de conjunto que pueden tener causa en las maneras que se relacionan los deportistas. Esto confirma que la particularidad de cada deporte conlleva su forma de crear cohesión, deseo de participación, motivación y sentido de pertenencia, generando buenos índices por cada deporte; sin embargo, pone a prueba si hay una coherencia en el discurso de los entrenadores. En ese sentido, el entorno deportivo universitario debe estar apoyado en la psicología para un entrenamiento íntegro en el que se tenga en cuenta esas diferencias y así construir un ambiente óptimo para el buen desarrollo personal, emocional y claramente, deportivo.

\section{Referencias}

Arias, I., Cardoso, T., Aguirre, H. \& Arenas, J. (2016). Características psicológicas de rendimiento deportivo en deportes de conjunto. Psicogente, n19(35), 25-36.http://doi.org/10.17081/ psico.19.35.1206

Arquer, I. (1999). NTP 534. Carga mental de trabajo: Factores. INSHT. Recuperado de: https://goo.gl/Ge7cF7

Cárdenas, D; Conde, J y Perales, J. (2015). El papel de la carga mental en la planificación del entrenamiento deportivo. Revista de Psicología del deporte [en línea]. Disponible en: http://www.redalyc. org/articulo.oa?id=235139639011 ISSN1132-239X.

Casarico, G. Motivando a un equipo. Publicado en página virtual en: http://www.psicologosdeldeporte.com/art_motivando.html 
Díaz, C. (2010). Actividad Laboral y Carga Mental de Trabajo. Ciencia \& Trabajo. Abr ,Jun; 12 (36): 281-292.

García, A. \& Ortiz, P. (2016) El sentido Psicológico del compromiso deportivo. Psicodeportes - Revista Online de la Asociación de Psicólogos del Deporte, octubre 2016 No. 23.

López, M. (2011). Memoria de trabajo y aprendizaje: Aportes de la neuropsicología. Cuaderno neuropsicológico. 5 (1) 25 - 47. Universidad Católica Argentina. Buenos Aires 239-3100 Paraná.

Nieto, G. \& Olmedilla, A. (2001) Planificación del entrenamiento psicológico en atletas de élite: un caso en marcha atlética. Revista de Psicología del Deporte 2001. 10, 1, 127-142

Serrato, L. (2008) Historia de la psicología del deporte en Colombia. Revista Iberoamericana de Psicología del Ejercicio y el Deporte, 3, 2, 277-300.

Pons, I. D., \& Puig, R. F. (2004). Revisión del concepto de carga mental: evaluación, consecuencias y proceso de normalización. Anuario de psicología/The UB Journal of psychology, 35(4), 521-546. 


\section{Anexos}

Índice de evaluación de NASA-TLX

\begin{tabular}{|l|l|}
\hline Puntaje & Interpretación \\
\hline Puntaje menor a 500 & Carga mental baja \\
\hline Puntaje entre 501 y 999 & Carga mental media \\
\hline Puntaje mayor a 1000 & Carga mental alta \\
\hline
\end{tabular}

Escalas de evaluación de CPRD

\begin{tabular}{|l|l|l|l|l|l|}
\hline Escalas & $\begin{array}{l}\text { Puntuación } \\
\text { máxima }\end{array}$ & $\begin{array}{l}\text { Puntuación } \\
\text { mínima }\end{array}$ & Media & $\begin{array}{l}\text { Desviación } \\
\text { típica }\end{array}$ & Alfa \\
\hline $\begin{array}{l}\text { Control de } \\
\text { estrés }\end{array}$ & 80 & 4 & 46,16 & 14,44 & 0,88 \\
\hline $\begin{array}{l}\text { Influencia } \\
\text { de la } \\
\text { evaluación } \\
\text { del } \\
\text { rendimiento }\end{array}$ & 45 & 3 & 25,11 & 7,63 & 0,72 \\
\hline Motivación & 31 & 7 & 19,59 & 5,066 & 0,67 \\
\hline $\begin{array}{l}\text { Habilidad } \\
\text { mental }\end{array}$ & 34 & 8 & 21,16 & 4,6 & 0,34 \\
\hline $\begin{array}{l}\text { Cohesión de } \\
\text { equipo }\end{array}$ & 24 & 0 & 18,48 & 4,31 & 0,78 \\
\hline
\end{tabular}

